\title{
Time and Frequency Localized Pulse Shape for Resolution Enhancement in STFT-BOTDR
}

\author{
Linqing Luo, ${ }^{1}$ Bo Li, ${ }^{1}$ Yifei Yu, $^{1}$ Xiaomin Xu, ${ }^{1}$ Kenichi Soga, ${ }^{1}$ and Jize Yan $^{1,2}$ \\ ${ }^{1}$ Department of Engineering, University of Cambridge, Trumpington Street, Cambridge CB2 1PZ, UK \\ ${ }^{2}$ Electronics and Computer Science, University of Southampton, Southampton SO17 1BJ, UK
}

Correspondence should be addressed to Jize Yan; yanjize@gmail.com

Received 2 September 2015; Revised 12 November 2015; Accepted 13 December 2015

Academic Editor: Marco Consales

Copyright (C) 2016 Linqing Luo et al. This is an open access article distributed under the Creative Commons Attribution License, which permits unrestricted use, distribution, and reproduction in any medium, provided the original work is properly cited.

\begin{abstract}
Short-Time Fourier Transform-Brillouin Optical Time-Domain Reflectometry (STFT-BOTDR) implements STFT over the full frequency spectrum to measure the distributed temperature and strain along the optic fiber, providing new research advances in dynamic distributed sensing. The spatial and frequency resolution of the dynamic sensing are limited by the Signal to Noise Ratio (SNR) and the Time-Frequency ( $T-F)$ localization of the input pulse shape. T-F localization is fundamentally important for the communication system, which suppresses interchannel interference (ICI) and intersymbol interference (ISI) to improve the transmission quality in multicarrier modulation (MCM). This paper demonstrates that the $T$-F localized input pulse shape can enhance the SNR and the spatial and frequency resolution in STFT-BOTDR. Simulation and experiments of T-F localized different pulses shapes are conducted to compare the limitation of the system resolution. The result indicates that rectangular pulse should be selected to optimize the spatial resolution and Lorentzian pulse could be chosen to optimize the frequency resolution, while Gaussian shape pulse can be used in general applications for its balanced performance in both spatial and frequency resolution. Meanwhile, T-F localization is proved to be useful in the pulse shape selection for system resolution optimization.
\end{abstract}

\section{Introduction}

Dynamic strain measurement in distributed fiber optic sensing (DFOS) is essential for structural health monitoring (SHM) of the strain changes induced by aging material and seismic or man-made activities [1]. Since the first system was launched in 1999, due to its stability, reliability, and insensitivity to external perturbations, the distributed Brillouin sensor has become one of the most popular diagnostic tools for SHM in bridges, dams, pipelines, and piles [2]. Among the different DFOS systems, BOTDR launches pulsed light into one end of the fiber and detects the scattered spontaneous Brillouin signal at the same end $[3,4]$. Single-end detection is convenient for fiber installation and on-site measurement. However, the large averaging number for a good Signal to Noise Ratio (SNR) and the slow frequency scanning method used in classical BOTDR have become obstacles to achieving dynamic measurement [5].

Recently, a fast-speed Short-Time Fourier Transform (STFT) based BOTDR applies a wideband detection architecture to replace the time-consuming frequency scanning method, which provides an opportunity for dynamic measurement. In the literature, the system successfully achieved a $16.7 \mathrm{~Hz}$ strain variation on a $12 \mathrm{~m}$ fiber section with $4 \mathrm{~m}$ spatial resolution and $45 \mu \varepsilon$ strain uncertainty [6]. The spatial resolution is the minimum detectable length of strain change and has been studied over the past decades to detect small cracks [7], while the strain uncertainty is the strain resolution for the measurement. A longer pulse or a longer rising/falling time will increase the interaction of the light photons and acoustic phonons and lead to a worse spatial resolution along the fiber [1].

BOTDR measures the peak frequency shift of the Brillouin scattering spectrum. SNR is the detective power density at spectrum's peak divided by the power of the whole spectrum including the sidebands. The SNR of the detected Brillouin scattering spectrum at each point along the fiber reduces when the sideband of the pulse's spectrum increases. Increased SNR will reduce average time of the measurement to enhance the speed of the analyzer to achieve real-site 
dynamic measurement [8]. SNR can be affected by the pulse power [9] and the pulse shape, which has significant impact on the spatial resolution, temperature, and strain resolution of the system [10-12].

Previous literatures compared time-domain and frequency-domain analysis independently to evaluate the analyzer performance. In 1999, Naruse and Tateda published the relation between the pulse width and the Brillouin backscattered light power and mentioned the trade-off between spatial resolution and frequency resolution [13]. In 2000, the same group announced that different pulse shapes would have different bandwidth of the spectrum and have different frequency-measurement error according to simulations [12]. Hao et al. in 2013 compared three pulse shapes including trapezoidal, triangular, and rectangular pulses and expressed that the Brillouin spectrum power of the triangular pulse shape was higher than the power of other pulse shapes $[10,14]$. They also compared other pulse shapes, including Lorentzian, Gaussian, super Gaussian, and triangular shape, and concluded that the Lorentzian shape generated the biggest peak power of Brillouin spectrum and has the measurement accuracy when the Brillouin spectrum peak is set to the same level [11].

In the above previous studies, assumptions were made that the spatial resolution was identical to the pulse width (full width at half maximum (FWHM) of the pulse) and the Brillouin spectrum was affected by frequency-domain information. Spatial resolution can be enhanced by reducing the pulse edges (in time-domain) and frequency resolution can be enhanced by reducing the sideband in pulse spectrum (in frequency-domain). However, time-domain and frequencydomain information are identically important, which should be considered simultaneously to offer the design tradeoff analysis between pulse width and frequency linewidth. For instance, when the frequency shifts (due to strain or temperature variation) are narrower than the FWHM, the spectrum will have double peaks and lead to complication in comparison [15], in which time- and frequency-domains' analysis needs to be considered cooperatively.

The Time-Frequency ( $T-F)$ localization has been utilized in communication and signal processing [16-18], which generates sharp localization, offers small distortion, and reduces the interference such as intercarrier interference (ICI) and intersymbol interference (ISI) in OFDM communication. The resolution in frequency and time-domain in image processing and computer visions can be enhanced in real applications using T-F localization [19-22].

We introduce the T-F localization into the design consideration of the BOTDR system. In this paper, TimeDomain Effective-Pulse Width (TEW) and FrequencyDomain Effective-Pulse Linewidth (FEL) are defined to offer a comprehensive view to optimize the analyzer performance. Different pulses with the same FWHM may have different TEW and/or different SNR requirement in the detection and the FEL can be different. In particular, the variance in laser output power, noise level, or averaging number, and so forth, in the same pulse shape, can result in different spatial and frequency resolution. Similarly, the pulses with various effective linewidth can lead to different noise immune ability in the system.

This paper demonstrates the importance for T-F localized input pulses to improve the SNR of the Brillouin scattering signal, which leads to a narrower frequency bandwidth and an efficient pulse length to enhance the spatial and frequency resolution. The pulse localization model, based on the physical description of BOTDR and time-frequency analysis of the pulses with the definition of TEW and FEL, has been built to investigate the performance of various pulse shapes, such as Lorentzian, rectangular, Gaussian, and triangular pulses, which are compared theoretically and experimentally according to their performances in the timedomain (spatial resolution) and the frequency-domain (pulse modulated Brillouin spectrum linewidth). The comparison of the four pulse shapes offered matched results in simulation and experiment in the same system and improved the understanding of detection of minimum frequency-changelength and frequency accuracy in different pulse shapes with the same FWHM. Hence the Gaussian pulse can be used in general measurement because of its balanced time and frequency resolution. Practically, the combination of different pulse shapes can be used to obtain accurate measurements in time- and frequency-domain, respectively. To enhance the analyzer performance, the methodology of the T-F localization method is a useful tool to design, compare, and implement the pulse and algorithm selection in DFOS. T-F localization should be also considered in general to evaluate the power, averaging number settings in DFOS development.

\section{Physical and Mathematical Description on BOTDR}

In a single mode fiber, $1550 \mathrm{~nm}$ light will generate Brillouin scattering signals whose peak frequency shifts about $11 \mathrm{GHz}$, due to the interaction between the lightwave and acoustic waves in the fiber $[23,24]$. The acoustic wave makes the Brillouin gain spectrum (BGS), a Lorentzian shape distribution whose linewidth is determined by the phonon lifetime [25]. Distributed fiber sensing systems detect the frequency shift of the BGS by searching the frequency peaks over time, hence producing distributed sensing information (temperature and strain) along the fiber $[26,27]$. The physical and mathematical model has been discussed by several research groups. Minardo et al. [28] constructed the solution of the BOTDA model, while Naruse and Tateda [13] offered a simplified BOTDR model, and Nishiguchi et al. [29] introduced a more general BOTDR model.

The Brillouin scattering process can be expressed by the following equations [25]:

$$
\begin{aligned}
\nabla^{2} \mathbf{E}_{\mathbf{p}}(\mathbf{r}, t)+\frac{n^{2}}{c^{2}} \frac{\partial^{2} E_{p}(r, t)}{\partial t^{2}} & =-\mu_{0} \frac{\partial^{2} \mathbf{P}_{\mathbf{p}}(r, t)}{\partial t^{2}}, \\
\nabla^{2} \mathbf{E}_{\mathbf{S}}(r, t)+\frac{n^{2}}{c^{2}} \frac{\partial^{2} \mathbf{E}_{\mathbf{S}}(r, t)}{\partial t^{2}} & =-\mu_{0} \frac{\partial^{2} \mathbf{P}_{\mathbf{S}}(r, t)}{\partial t^{2}}, \\
\frac{\partial^{2} \Delta Q}{\partial t^{2}}-\Gamma \nabla^{2} \frac{\partial \Delta Q}{\partial t}-v_{a}^{2} \nabla^{2} \Delta Q & =-\nabla \mathbf{f},
\end{aligned}
$$


where $E_{p}, E_{S}$, and $Q$ are the amplitudes of the pump light, stokes light, and acoustic wave, respectively. $P_{p}$ and $P_{S}$ are the polarization density of the pump and stoke waves, respectively. $f$ is the electrostrictive force per unit volume.

By simplifying the equation and assuming that noise is constant along the fiber [28-30],

$$
\begin{aligned}
\left(\frac{\partial}{\partial z}+\frac{n}{c} \frac{\partial}{\partial t}+\frac{\alpha}{2}\right) E_{p} & =-Q E_{S}, \\
\left(-\frac{\partial}{\partial z}+\frac{n}{c} \frac{\partial}{\partial t}+\frac{\alpha}{2}\right) E_{S} & =Q^{*} E_{p}, \\
\left(\frac{\partial}{\partial t}+\Gamma\right) Q & =i g_{B} E_{p} E_{S}^{*}+R(z, t),
\end{aligned}
$$

where $c$ is the light velocity in a vacuum, $n$ is the refractive index of the fiber, $\Gamma$ is the sum of the damping of the phonon lifetime, and the detuning parameter $\Gamma=\Gamma_{B} / 2+i S_{B}(z) . S_{B}(z)$ is the frequency shift generated by Brillouin scattering, which is proportional to the independent thermodynamic variables of the entropy, $T$, and pressure, $p$ :

$$
S_{B}(z)=\left(\frac{\partial S_{B}}{\partial p}\right)_{s} \Delta p+\left(\frac{\partial S_{B}}{\partial p}\right)_{p} \Delta s .
$$

Because Spontaneous Brillouin Scattering (SpBS) does not have a stimulated effect, the SBS terms in (2) and (4) are assumed to be zero to simplify the analysis.

Then the scattered light can be expressed as

$$
\begin{gathered}
E_{S}(t, 0)=i \sqrt{P_{P} / A_{\mathrm{eff}}} \kappa e^{-(\alpha / 2) v_{g} t} \int_{0}^{L} f\left(t-\frac{2 z}{v_{g}}\right) \\
\cdot Q\left(t-\frac{z}{v_{g}}, z\right) d z .
\end{gathered}
$$

The spectrum can be described as

$$
H(t, \omega)=\xi \int_{-\infty}^{+\infty} \int_{0}^{L} f\left(t-\frac{2 z}{v_{g}}\right) Q(t, z) e^{-i \omega t} d z d t .
$$

This can be written in convolution form as [29]

$$
\begin{aligned}
H(t, \omega) & =\gamma[L(t, \omega) * \Psi(t, \omega)], \\
L(t, \omega) & =\frac{(\Gamma / 2)^{2}}{(\Gamma / 2)^{2}+\left(\omega-S_{B}\left(v_{g} t / 2\right)\right)^{2}} \\
\Psi(t, \omega) & =F_{t}(f(t)),
\end{aligned}
$$

where $\gamma$ is the time-nondependent coefficient and $\Psi(t, \omega)$ is the Fourier transform of the pulse signal $f(t)$.

At a particular time, according to the model given by Naruse and Tateda [13], the spectrum of the Brillouin scattered signal at each point along the fiber can be expressed as

$$
H(\nu)=\int_{-\infty}^{+\infty} P_{p}\left(f, f_{0}\right) \frac{h(\omega / 2)^{2}}{\left[\nu-\left(f-S_{B}\right)^{2}\right]+(\omega / 2)^{2}} d f,
$$

where $P_{p}\left(f, f_{0}\right)$ is the power spectrum of a launched pulse in which each frequency component produces a Lorentzian shape Brillouin backscattered light spectrum profile with peak frequency of $f-S_{B}$ and full width at half maximum (FWHM) of $\omega$. The term $S_{B}$ expresses the frequency changes due to local acoustic waves, where the difference comes from the changes in the properties of the fiber or the changes in strain or temperature.

Based on the equations above, the final scattered back Brillouin spectrum is the integration of all the spectra generated by each frequency component on the pump pulse spectrum. According to (8) and (11), the time-varying Brillouin spectrum is related to the entire pulse shape. In the pulse's rising and falling edges, the spatial resolution will be reduced because the power in the edges will worsen the spatial resolution by expanding the length of wave interaction. Hence, time localized pulse can enhance the spatial resolution.

The broadened bandwidth and expanded sideband increase the complexity of accurate peak frequency detection. The noise will be increased when the sideband power is comparable with mainlobe power in the pulse modulated SpBS spectrum [12]. In order to remain a good SNR, the sidebands' power should be minimized. Therefore the frequency localized pulses in BOTDR require the power to be concentrated near the peak frequency to maintain a narrow linewidth.

\section{Time-Frequency $(T-F)$ Localization}

The mean square deviation of the time and frequency distribution of a signal is defined in (12) and (13) [16]. Equation (14) describes the Heisenberg uncertainty principle [17]:

$$
\begin{aligned}
\Delta T^{2} & =\int_{-\infty}^{\infty} t^{2}\left|f(t)^{2}\right| d t, \\
\Delta F^{2} & =\int_{-\infty}^{\infty} f^{2}\left|F(f)^{2}\right| d f, \\
\Delta T^{2} \Delta F^{2} & \geq \frac{1}{4},
\end{aligned}
$$

where $\Delta T$ and $\Delta F$ are the variance of the signal $f$ in the timeand frequency-domains, respectively.

In the telecommunication systems, the dispersion of the signal causes intersymbol and interchannel interference when the signal has multicarriers, such as in an Orthogonal Frequency Division Multiplex (OFDM) system [31]. When the signal disperses over time, it may also interfere with subsequent symbols to cause "intersymbol" interference (ISI), reducing the reliability of the communications system [18]. When the signal disperses in the frequency-domains, it may interfere with symbols in other channels, causing "interchannel" interference (ICI), reducing the SNR in each channel [19]. A delta function in the frequency-domain produces a continuous single frequency lightwave with constant power. The uncertainty principle governs that delta functions in both time- and frequency-domains cannot simultaneously exist in the same signal. 
TABLE 1: Variation in the time- and frequency-domains with the product of the two variations for the pulse shapes.

\begin{tabular}{lcccc}
\hline & Rectangular & Lorentzian & Gaussian & Triangular \\
\hline$\Delta T$ & $2.60 e-13$ & $5.75 e-13$ & $2.12 e-13$ & $2.08 e-13$ \\
$\Delta F$ & $3.51 e+17$ & $6.88 e 13$ & $1.87 e 14$ & $2.03 e 14$ \\
$\Delta T \times \Delta F$ & 91457.02 & 39.54 & 39.58 & 42.24 \\
\hline
\end{tabular}

$T-F$ localization describes the extent to which a pulse signal is restricted in both the time- and frequency-domains. Good localization describes the minimum energy spreading over a certain time span and frequency bandwidth to avoid the power leakage into channels and interfering with neighboring symbols [32]. Besides in telecommunication, the $T-F$ localization is also well studied in high resolution geophysical data analysis [20] and in wavelets transform in signal processing [21].

Pulse shapes have different localization characteristic. Rectangular, Lorentzian, Gaussian, and triangular shape pulses were simulated in 3D using STFT. In order to plot the time- and frequency-domain of the pulses simultaneously, the pulses were multiplied with a square STFT window to do the time-frequency analysis of the shapes. The STFT 3D plots and the sideband in both domains are shown in Figures 1 and 2. The pulses all had $50 \mathrm{~ns}$ FWHM and $1 \mu$ s period and were analyzed using $50 \mathrm{~ns}$ window STFT.

The variances obtained by (12) and (13) in time- and frequency-domain for different pulse shapes and the product of the variances are given in Table 1 . The Lorentzian shape has the smallest product with the same FWHM of the normalized pulses, showing that the Lorentz shape performs the best in both time and frequency localization, using the traditional telecommunication definition.

\section{Time-Frequency Localization in the BOTDR System}

The product of $\Delta T \times \Delta F$ is a measure of the performance of a pulse, where $\Delta T$ and $\Delta F$ are defined as the variance of the pulse in the time-domain and frequency-domain, respectively. In a Brillouin optic fiber sensing system, the target is no longer to reduce the ISI and ICI in channels but to enhance the efficient pulse length and narrow the linewidth of the pulse modulated Brillouin spectrum, providing a better SNR in time- and frequency-domains, so that the system can achieve long distance and high resolution measurement.

A pulse consists of two parts in time-domain, mainlobe, and tails. Conventionally, the mainlobe is the power within the FWHM width and the tails are the parts of rising and falling edges. The final pulse modulated Brillouin spectrum consists of two parts of Brillouin scattering in the fiber: one generated by the mainlobe part of the pulse and one generated by the tails of the pulse.

When a uniform strain and temperature are applied to a fiber, the center frequencies of the SpBS remain constant; thus, the tails in pulse do not affect the monitoring result. When strain or temperature changes, the frequency of SpBS
TABLE 2: Extinction ratios of the four normalized shapes with $50 \mathrm{~ns}$ FWHM.

\begin{tabular}{lcccc}
\hline & Rectangular & Lorentzian & Gaussian & Triangular \\
\hline $\begin{array}{l}\text { Extinction in } \\
\mathrm{dB}\end{array}$ & 55.92 & 0.55 & 10.01 & 9.50 \\
\hline
\end{tabular}

changes, and Brillouin scattering generated by mainlobe and tails are different. If the changes happen in the tails' part, the changes will affect the result of the mainlobe part. If the power in the tail is comparable to that in the mainlobe, the peaks of the two parts will be apparent from each other, reducing the frequency accuracy due to the double peak effect. Hence, the tail of pulses will reduce the spatial resolution.

In the pulses plotted in Figure 3, the power in the mainlobe, shown in red, is $P_{S}$ and the powers in the two tails, shown in blue, are $P_{T 1}$ and $P_{T 2}$ and are assumed to be the same, as $P_{T}=P_{T 1}=P_{T 2}$. The width of mainlobe is the FWHM of the pulse. The extinction ratio is defined as

$$
\begin{aligned}
\text { Extinction ratio } & =\frac{\text { the power existing in the area }}{\text { The power in the tail }} \\
& =\frac{P_{\text {sig }}}{2 P_{T}} .
\end{aligned}
$$

The extinction ratios of the different pulse shapes are summarized in Table 2.

In these shapes, a rectangular pulse has ideal edges in time-domain; however, in the frequency-domain, the sideband is the worst, while the Lorentzian pulse performs best but is worst in the time-domain. The Lorentzian shape has the smallest product of pulse width and frequency bandwidth in Table 1 but has the lowest extinction ratio in Table 2. The Gaussian and triangular shape have similar extinction ratio which is worse than Lorentzian shape and better than rectangular shape. The requirements for the extinction ratio and the system resolution can differ from the pulse width with the different signal processing methods $[33,34]$, but the $T-F$ localization can indicate the effectiveness of the input pulses in relation to the spatial and frequency resolution in BOTDR systems.

This paper defines two parameters for BOTDR, one in time-domain-the Time-Domain Effective-Pulse Width (TEW) - and one in frequency-domain - the FrequencyDomain Effective-Pulse Linewidth (FEL). TEW determines the effective spatial resolution detected by the monitoring system. FEL is the actual spectral linewidth required to obtain a required spectrum SNR for peak detection. The mainlobe in time-domain is defined as the part of the pulse that lies within the TEW, and the tails are defined as the parts of the pulse outside TEW in time-domain. The mainlobe in frequency-domain is the frequency slots within FEL while the sideband is formed from the frequency slots outside the FEL in frequency-domain.

In the time-domain, $\alpha$ is defined as the ratio of the power in the mainlobe to that in the tails of the pulse. If the detection system requires $\alpha$ (unit in $\mathrm{dB}$ ) to obtain the precise frequency shift information, the effective pulse width is that which 


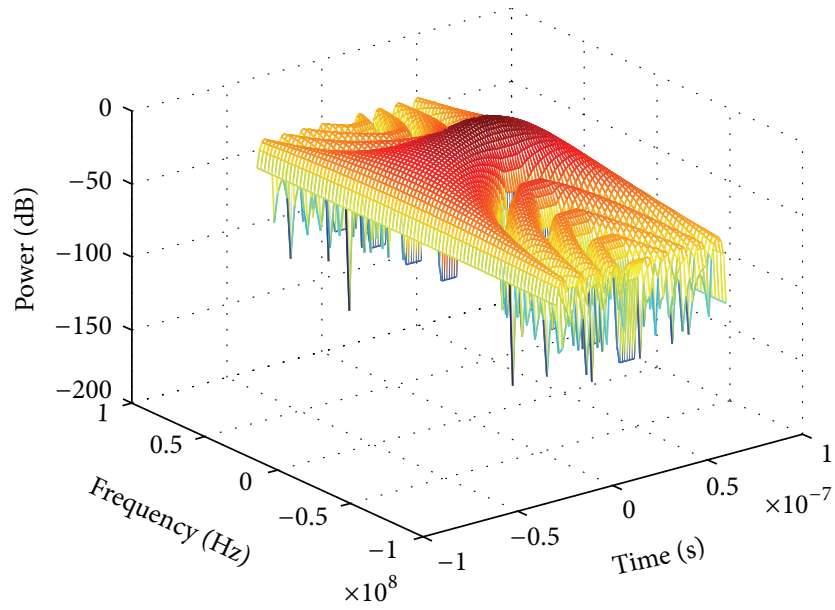

(a)

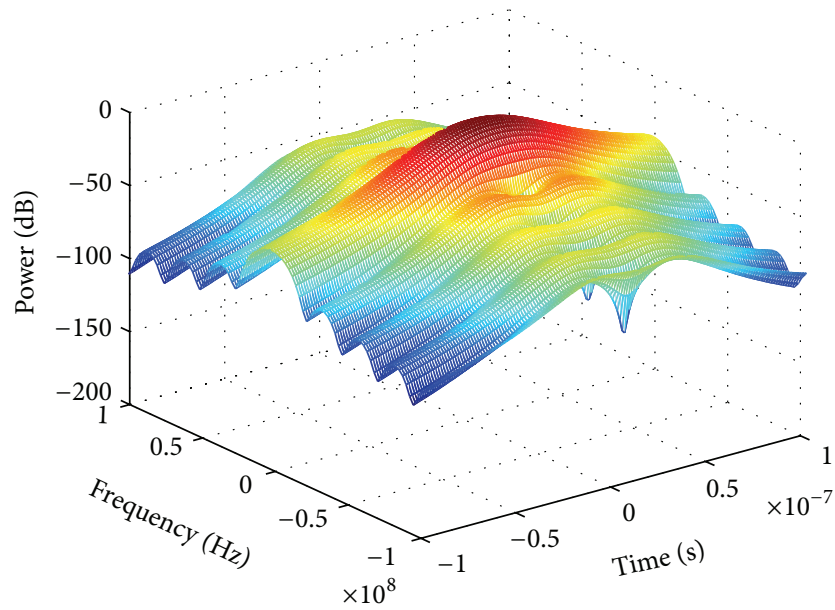

(c)

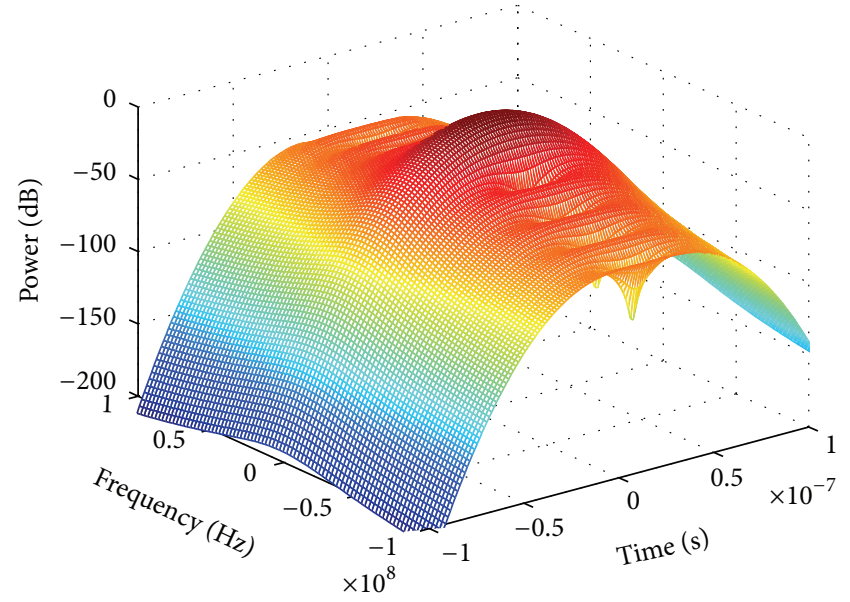

(b)

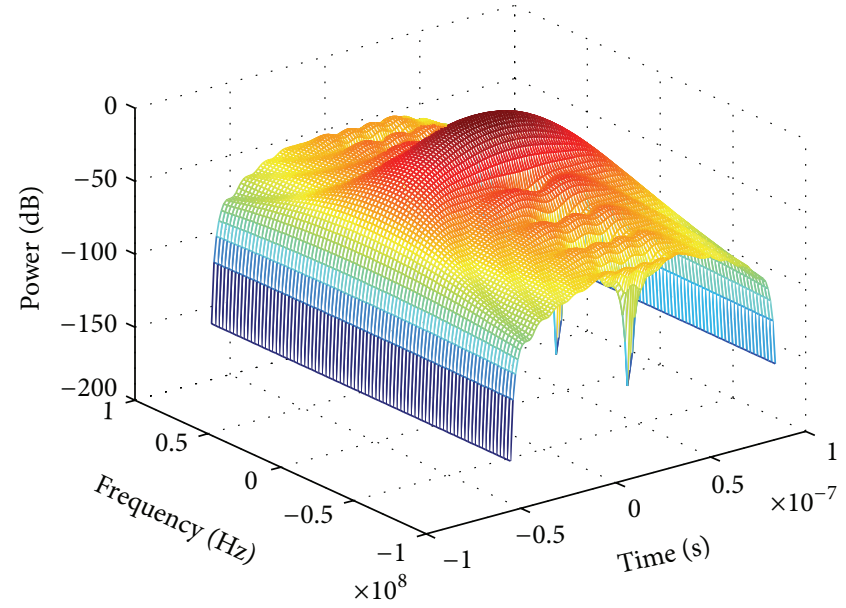

(d)

FIGURE 1: 3D plots of $50 \mathrm{~ns}$ FWHM pulses in the time- and frequency-domains. (a) STFT plot of rectangular pulse; (b) STFT plot of Gaussian pulse; (c) STFT plot of Lorentzian pulse; (d) STFT plot of triangular pulse.

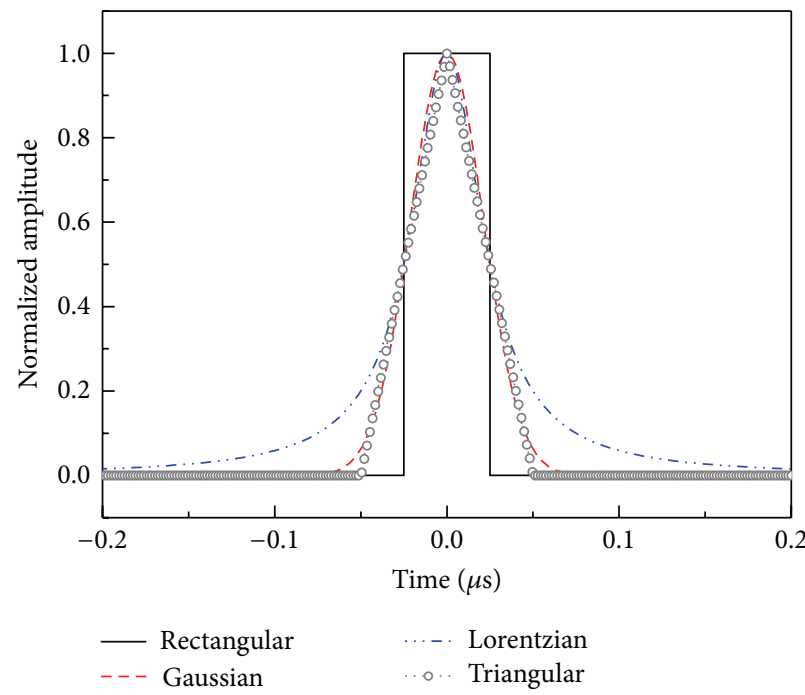

(a)

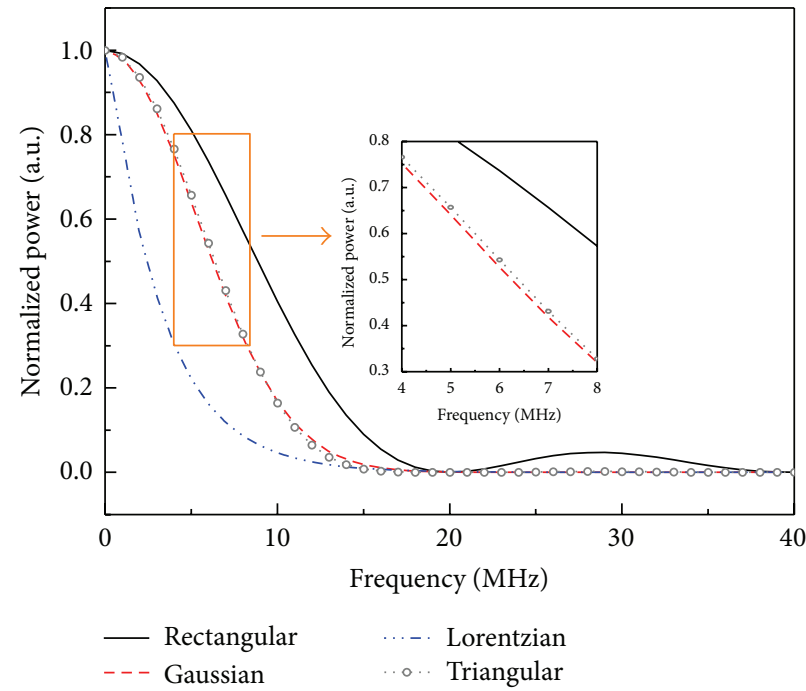

(b)

Figure 2: (a) Pulses in the time-domain. (b) Pulses in the frequency-domain. 


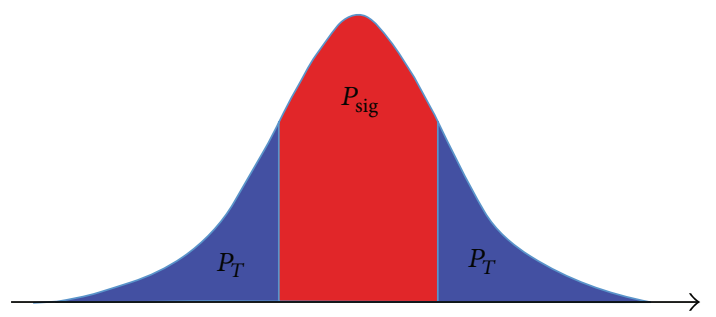

FIGURE 3: The comparative power of the mainlobe and tails in a pulse shape.

TABLE 3: Effective pulse width and the corresponding effective spatial resolution for the four pulse shapes.

\begin{tabular}{lcccc}
\hline & Rectangular & Lorentzian & Gaussian & Triangular \\
\hline $\begin{array}{l}\text { TEW (ns) } \\
\begin{array}{l}\text { Physical } \\
\text { spatial }\end{array}\end{array}$ & 33.2 & 80.2 & 41 & 42.1 \\
$\begin{array}{l}\text { resolution } \\
(\mathrm{m})\end{array}$ & 3.32 & 8.02 & 4.1 & 4.21 \\
\hline
\end{tabular}

satisfies (16); and it can be written in the integration form as in (17):

$$
\begin{aligned}
P_{S}-2 P_{T} & =\alpha \quad(\text { in } \mathrm{dB}) \\
10 \log \frac{\int_{-\Delta T_{\alpha} / 2}^{\Delta T_{\alpha} / 2} f(t) d t}{2 \times \int_{\Delta T_{\alpha} / 2}^{\infty} f(t) d t} & =\alpha,
\end{aligned}
$$

where $f(t)$ is the normalized distribution of the shape and $\Delta T_{\alpha}$ is the TEW.

Let us assume that, for the plotted pulses with $50 \mathrm{~ns}$ FWHM width, the system can only detect the accurate peak when the pulse's mainlobe power is $3 \mathrm{~dB}$ greater than the tail's power. The TEW of the plotted pulses is shown in Table 3. With the same FWHM designed width, the TEW of the Lorentzian shape is more than twice as large as the rectangular pulse. The TEW of Gaussian and triangular shape is slightly wider than rectangular but still much smaller than Lorentzian shape, showing that the rectangular shapes will give the best spatial resolution.

In the frequency-domain, FEL is defined as if the system requires that the power of the spectrum peak is $\beta \mathrm{dB}$ higher than the noise level; thus, the effective Brillouin linewidth is the "bandwidth" at $\beta \mathrm{dB}$ lower than the spectrum peak. Because the power level of the Brillouin spectrum is proportionate to the power of the pulse spectrum, the FEL of the pulse is the bandwidth of the power level that is $\beta \mathrm{dB}$ lower than the peak level, as shown in Figure 4. Hence, it can be expressed by

$$
\begin{aligned}
P_{f l}-2 P_{f n} & =\beta \quad(\text { in } \mathrm{dB}) \\
10 \log \frac{\int_{-\Delta F_{\beta} / 2}^{\Delta F_{\beta} / 2} F(f) d f}{2 \times \int_{\Delta F_{\beta} / 2}^{\infty} F(f) d f} & =\beta,
\end{aligned}
$$

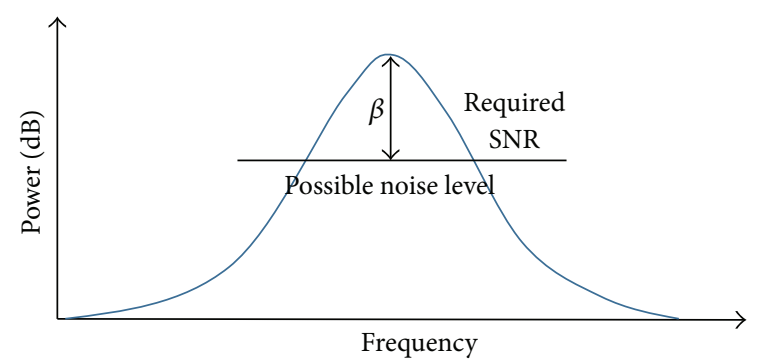

FIGURE 4: Plot of the pulse shape showing the definition of $\beta$, which is the difference between the peak power and the noise floor.

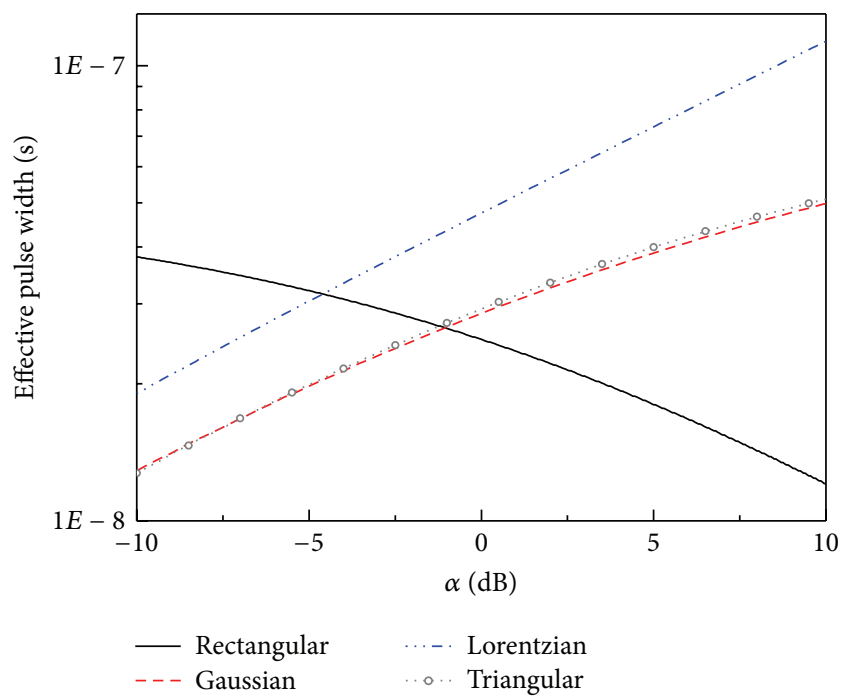

FIGURE 5: Changes of effective pulse width (TEW) with the ratio $\alpha$ changing from $-10 \mathrm{~dB}$ to $10 \mathrm{~dB}$ for different pulse shapes.

where $P_{f l}$ is the power included in the frequency mainlobe which has the width of FEL, $P_{f n}$ is the power in the sideband of the pulse's spectrum, and $\Delta F_{\beta}$ is the FEL of the pulses.

\section{Simulation and Experiment on Pulse Shapes}

Based on the definition of the Time-Frequency localization in the BOTDR system, the performance of Lorentzian, Gaussian, triangular, and rectangular pulse shapes will depend on $\alpha$ and $\beta$ values to quantify the spatial and frequency resolution. Linear variation of the $\alpha$ and $\beta$ values is simulated from $-10 \mathrm{~dB}$ (a noise-immune system) to $10 \mathrm{~dB}$ (a noise-sensitive system) to evaluate TEW and FEL. Rectangular, Lorentzian, Gaussian, and triangular shape input pulses with $50 \mathrm{~ns}$ pulse width and the same peak voltage are experimentally chosen to demonstrate and evaluate the T-F localization in the STFTBOTDR system.

5.1. Simulation Result. In STFT-BOTDR, $\alpha$ and $\beta$ need to be optimized to reduce $\Delta T_{\alpha}$ and $\Delta F_{\beta}$, improving the solution of the detecting system. Figures 5 and 6 show the changes in TEW and FEL when system $\alpha$ varies from $-10 \mathrm{~dB}$ to $10 \mathrm{~dB}$. The rectangular pulse has the best performance because it 


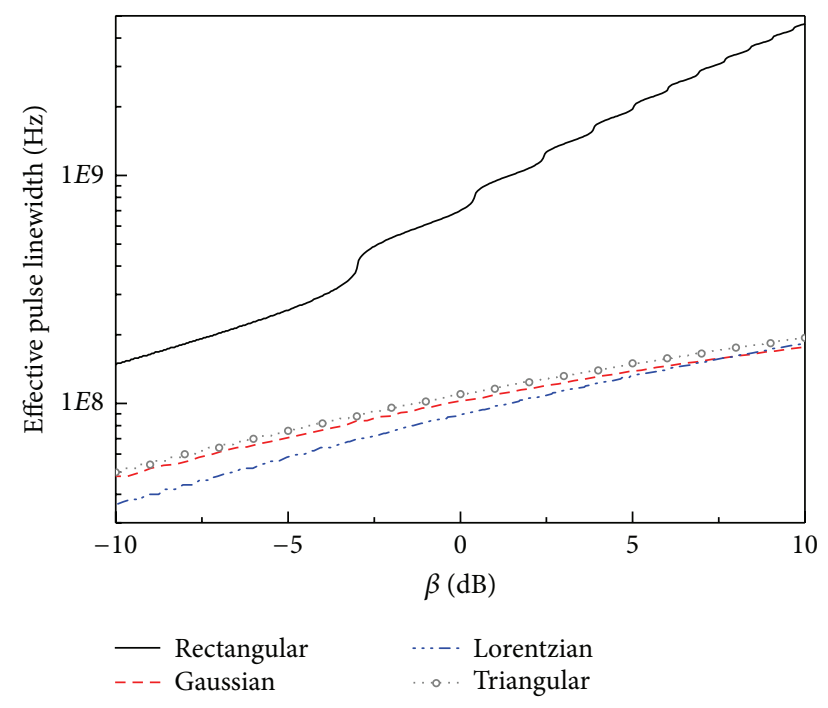

FIGURE 6: Changes in effective pulse linewidth (FEL) with the ratio $\beta$ changing from $-10 \mathrm{~dB}$ to $10 \mathrm{~dB}$ for different pulse shapes.

contains no tails but only the mainlobe signal. Other pulses show a rising trend when the system requires an increasing extinction ratio in the time-domain. Among those pulses, the Lorentzian shape pulse is the worst when $\alpha$ is large (which means that the system will have a larger spatial resolution with Lorentzian shape).

In the frequency-domain, the changes of FEL show opposite trends compared to the time-domain. The Lorentzian shape has the smallest linewidth and shows a slow increment with increasing $\beta$, whereas the rectangular pulse has the largest linewidth and expands rapidly when $\beta$ increases in the frequency-domain. In the two domains, the Gaussian and triangular pulse have similar performance which are always better than Lorentzian shape and worse than rectangular shape when $\alpha$ is larger than $0 \mathrm{~dB}$. The Gaussian shape is always slightly better than triangular shape though the result is very close.

Generally, $\alpha$ and $\beta$ should be minimized to enhance SNR and system resolution during system development. With a low SNR system, the Gaussian pulse gives a balanced performance in both the time- and frequency-domains, while others can have better performance in either spatial resolution or frequency resolution, sacrificing the other, too.

\subsection{Experimental Setup and Results. STFT-BOTDR using} frequency downconversion and STFT algorithm is shown in Figure 7. An ultra-narrow-line-width laser with the wavelength of $1554.4 \mathrm{~nm}$ was used as the light source. In branch A, after passing a coupler, a part of the continuous light was modulated by an electrooptic modulator (EOM) with a $20 \mu \mathrm{s}$ period and 50 ns pulse width signal generated by an Agilent 33600 arbitrary waveform generator (AWG). The pulsed light was amplified by an Erbium-doped fiber amplifier (EDFA) and then circulated into a long sensing fiber to generate the Brillouin backscattering signal. This signal was then heterodyned with the reference CW light in branch $\mathrm{B}$ and transferred to a RF signal using a wideband photodetector. The frequency of the signal was then downconverted to the intermediate frequency (IF) signal, which was then sampled in the time-domain and processed using the STFT algorithm to obtain the frequency-domain information-the SpBS spectrum along the fiber under test.

The fiber under test (FUT) was a $500 \mathrm{~m}$ single mode fiber with heating sections, immersed in a water bath at the end. The heating part is shown in Figure 8. The heating sections were of different lengths $5 \mathrm{~m}, 4 \mathrm{~m}, 3 \mathrm{~m}, 2 \mathrm{~m}, 1 \mathrm{~m}, 0.8 \mathrm{~m}$, and $0.5 \mathrm{~m}$. Each section was separated by a distance of $10 \mathrm{~m}$. All the sections were heated to $75^{\circ} \mathrm{C}$ and the rest of the fiber remained at room temperature.

Rectangular, Lorentzian, Gaussian, and triangular pulses, respectively, were experimentally generated as the source into the system, shown in Figure 9, to compare the performance in terms of the spatial resolution and pulse modulated Brillouin scattering spectrum linewidth at the same position along the testing fiber. Figure 10 shows the distribution of the center frequency along the fiber which measured the temperature distribution along the FUT. Conventionally, 50 ns pulses are considered to have a $5 \mathrm{~m}$ spatial resolution as the pulse width is about $50 \mathrm{~ns}[4,34]$. However, in the experimental results, all pulses could detect the spatial resolution lower than $5 \mathrm{~m}$ because the TEW of each pulse was less than the pulse width; hence the spatial resolution is smaller than the conventional understanding by using advanced signal processing algorithms. The Lorentzian, Gaussian, and triangular pulses could only detect the change in the $3 \mathrm{~m}$ section; however, the rectangular shape clearly monitored the temperature changes in the $2 \mathrm{~m}$ section, showing that the rectangular shape has a better TEW and gives an improved spatial resolution. In the center frequency plot, the Lorentzian shape shows a drop in $5 \mathrm{~m}$ section and center frequency detected decreases rapidly in small temperature change sections, given that its spatial resolution is the worst among the four shapes. The Gaussian pulse is slightly better than triangular shape with less reduction in center frequency in small sections though they both have similar peak detection in $5 \mathrm{~m}$ and $4 \mathrm{~m}$ section with rectangular pulse, but their detected center frequency deviates from correct value after $4 \mathrm{~m}$ section as well.

In the frequency-domain, shown in Figure 11, the rectangular pulse has a wider linewidth and a larger sideband, given the widest Brillouin scattering spectrum linewidth, which worsens the SNR of the peak power. The Lorentzian pulse performed the best, with the smallest FEL in the Brillouin spectrum. The Gaussian and triangular pulse again have middle FEL but they are close to Lorentzian pulse. When the changes are smaller or close to the spatial resolution, the Brillouin spectrum linewidth depends on the property of pulses not only in frequency-domain but also in the time-domain, because the overall frequency information over the detection range of the pulse will be compacted and represented in one localized spectrum. For instance, when the TEW of a pulse is large, such as Lorentzian shape, though it has smallest linewidth, its long tail in time-domain shared the frequency information over the entire measurement distance the pulse covered. Hence the final spectrum will include separate frequency shift components, causing the 


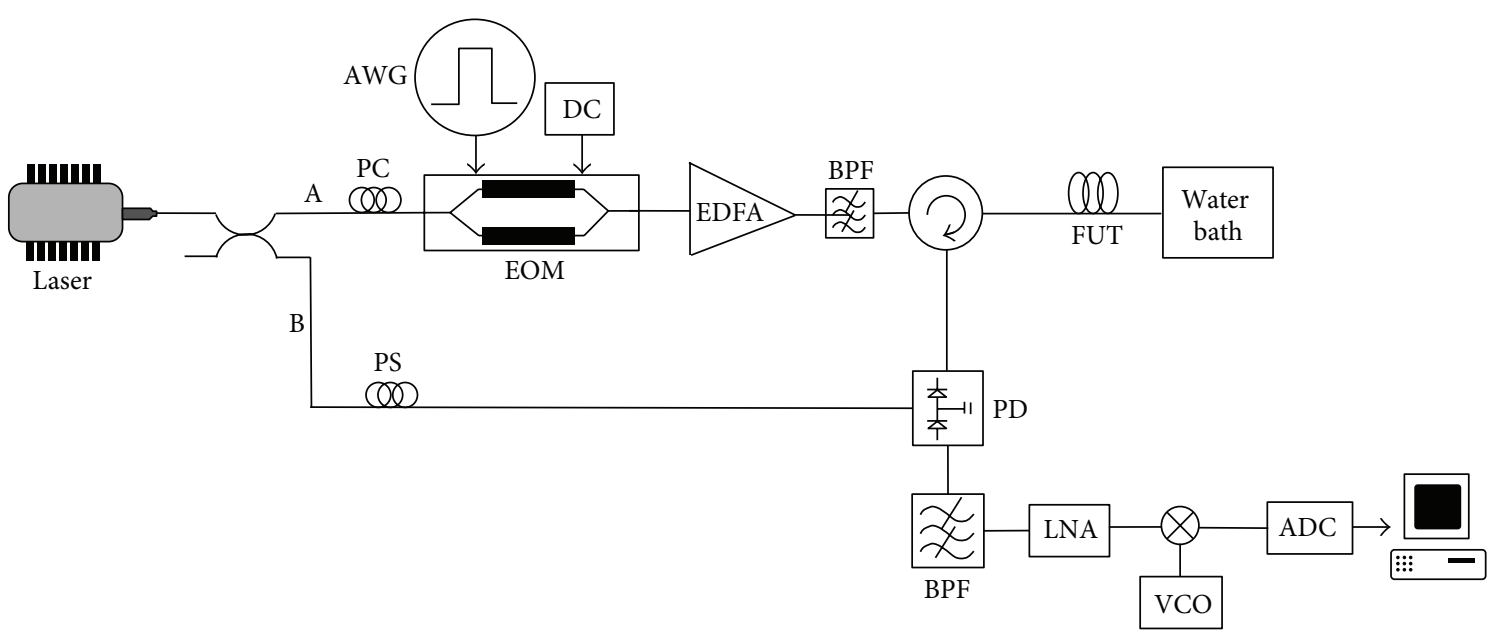

FIGURE 7: Architecture of STFT-BOTDR.

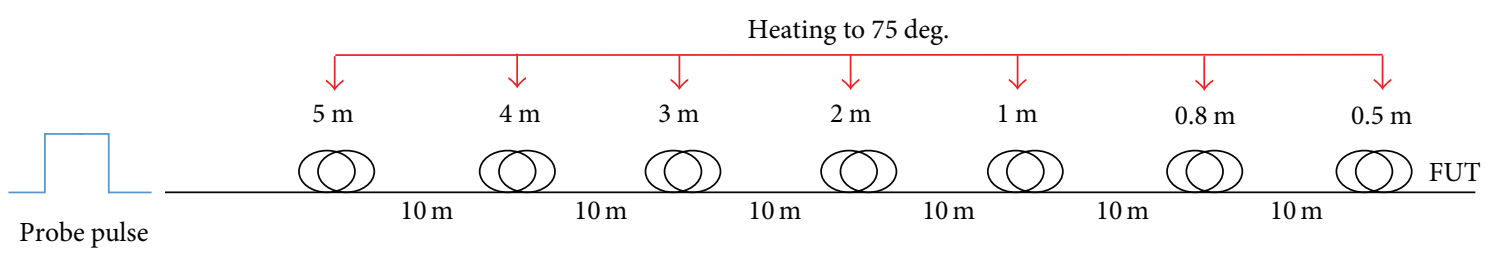

FIGURE 8: Heating part of FUT in the experiment with different heating lengths.

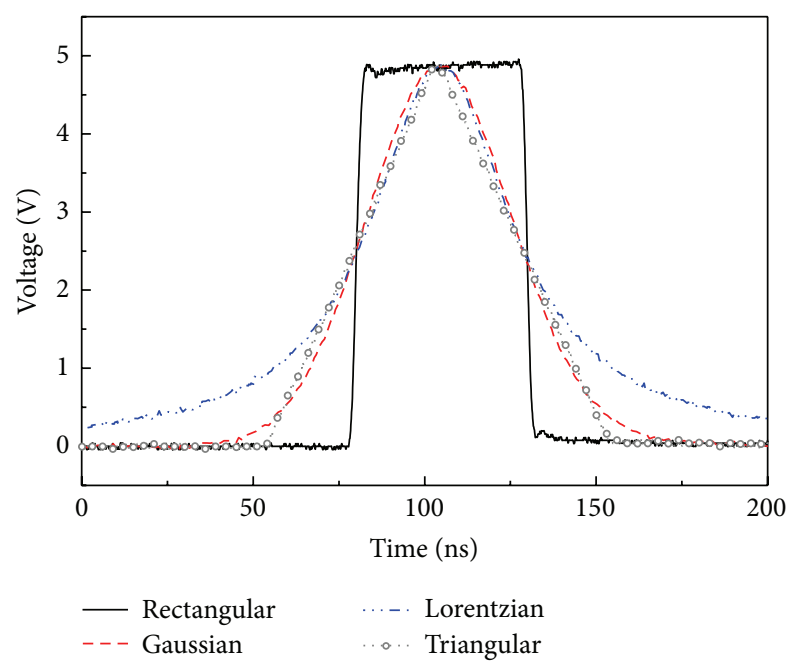

FIGURE 9: The pulses shape generated by AWG to use as modulation in $\mathrm{EOM}$.

double-peak effect. As a result, the Lorentzian pulse has an error in the center frequency detection over $5 \mathrm{~m}$ section.

Hence, with the same FWHM of the pulses, the rectangular pulse has the best spatial resolution, while the Lorentzian gives the best linewidth. The result shows that the rectangular pulse has better time localization and the Lorentzian shape has better frequency localization. The Gaussian and triangular shape have balance performance in two domains but Gaussian pulse is slightly better in the experiment.

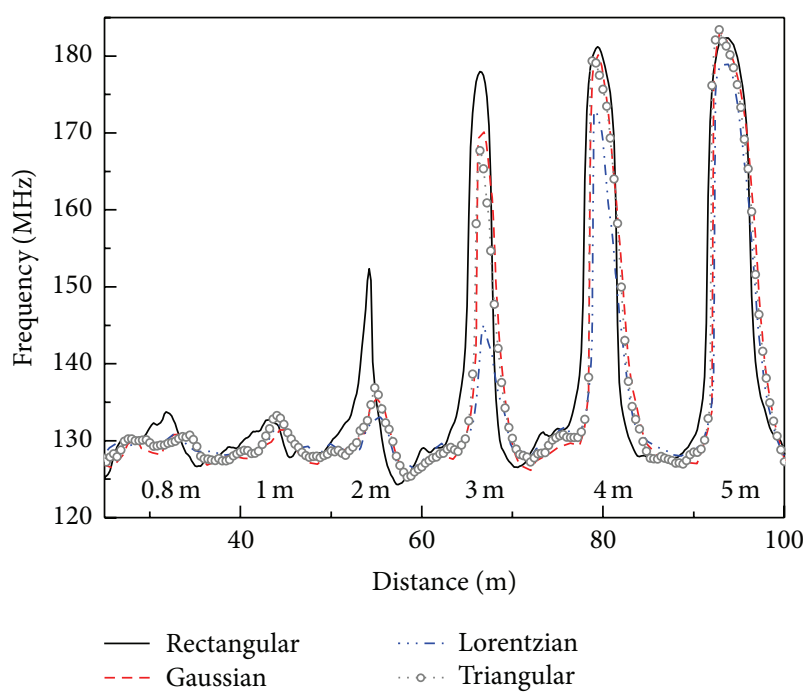

FIGURE 10: Peak center frequency distribution along the fiber after using 50 ns rectangular, Gaussian, triangular, and Lorentzian shape pulses.

\section{Conclusion}

In this paper, we discussed the importance of TimeFrequency $(T-F)$ localization and its contribution to improving spatial and frequency resolution. T-F localization in telecommunication systems was demonstrated and its alternative definition in the BOTDR system was introduced. 


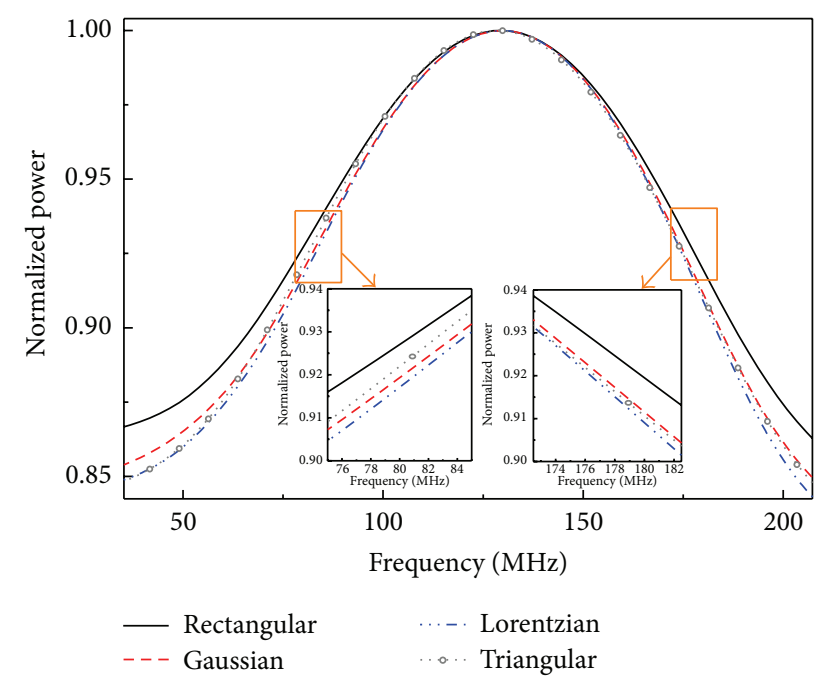

Figure 11: Pulse modulated Brillouin spectrum after using $50 \mathrm{~ns}$ rectangular, Gaussian, triangular, and Lorentzian shape pulses.

With the new definition of Time-Frequency localization in BOTDR, the Time-Domain Effective-Pulse Width (TEW) and Frequency-Domain Effective-Pulse Linewidth (FEL) were introduced as important parameters for resolution optimization. The conventional relationship of pulse width and spatial resolution is challenged with advanced architecture and signal processing method. Instead, TEW and FEL are more suitable to become a bridge between spatial resolution and frequency resolution and to describe the performance of a BOTDR system. Furthermore, rather merely one domain is considered in most of the papers, which is not suitable for real cases; the T-F localization method considers two domains simultaneously. Two parameters $\alpha$ and $\beta$ are defined to quantify the effect of TEW and FEL in BOTDR systems, which should be optimized to enhance the SNR and spatial and frequency resolution during system development. Commonly used input pulses, such as Lorentzian, rectangular, Gaussian, and triangular shape, were simulated and experimentally demonstrated in STFT-BOTDR to compare the Signal to Noise Ratio (SNR), the T-F localization, and the system resolution. The result indicates that the rectangular pulse should be selected when spatial resolution is crucial for the application and the Lorentzian shape should be chosen for the application requiring high frequency resolution. In practical usage, the two pulses could be combined in separate measurements to obtain a strain profile with optimized spatial and frequency resolution. Gaussian shape pulse has more balanced resolution in the time- and frequency-domains, which is suitable for general applications. The T-F localization is proved to be a powerful tool in the system design and development process to compare and implement the pulse shape, algorithm, and the selection and optimization of power, averaging number and relevant settings.

\section{Disclosure}

Following EPSRC policy on research data, the additional data will be accessible at https://www.repository.cam.ac.uk/ handle/1810/250413.

\section{Conflict of Interests}

The authors declare that there is no conflict of interests regarding the publication of this paper.

\section{Acknowledgments}

Linqing Luo and Bo Li are supported by the China Scholarship Council and Cambridge Commonwealth, European and International Trust. EPSRC EP/K000314/1 Grant is acknowledged.

\section{References}

[1] X. Bao and L. Chen, "Recent progress in Brillouin scattering based fiber sensors," Sensors, vol. 11, no. 4, pp. 4152-4187, 2011.

[2] M. DeMerchant, A. Brown, X. Bao, and T. Bremner, "Structural monitoring by use of a Brillouin distributed sensor," Applied Optics, vol. 38, no. 13, pp. 2755-2759, 1999.

[3] S. Uchida, E. Levenberg, and A. Klar, "On-specimen strain measurement with fiber optic distributed sensing," Measurement, vol. 60, pp. 104-113, 2014.

[4] F. Wang, X. Zhang, Y. Lu, R. Dou, and X. Bao, "Spatial resolution analysis for discrete Fourier transform-based Brillouin optical time domain reflectometry," Measurement Science and Technology, vol. 20, no. 2, Article ID 025202, 2009.

[5] Y. Lu, Y. Yao, X. Zhao, F. Wang, and X. Zhang, "Influence of nonperfect extinction ratio of electro-optic modulator on signalto-noise ratio of BOTDR," Optics Communications, vol. 297, pp. 48-54, 2013.

[6] G. Tu, X. Zhang, Y. Zhang, Z. Ying, and L. Lv, "Strain variation measurement with short-time Fourier transform-based Brillouin optical time-domain reflectometry sensing system," Electronics Letters, vol. 50, no. 22, pp. 1624-1626, 2014.

[7] C. A. Galindez-Jamioy and J. M. López-Higuera, "Brillouin distributed fiber sensors: an overview and applications," Journal of Sensors, vol. 2012, Article ID 204121, 17 pages, 2012.

[8] A. Funnell, X. Xu, J. Yan, and K. Soga, "Simulation of noise within BOTDA and COTDR system to study the impact on dynamic sensing," International Journal of Smart Sensing and Intelligent Systems, vol. 8, no. 3, pp. 1576-1600, 2015.

[9] C. N. Pannell, J. Dhliwayo, and D. J. Webb, “The accuracy of parameter estimation from noisy data, with application to resonance peak estimation in distributed Brillouin sensing," Measurement Science and Technology, vol. 9, no. 1, pp. 50-57, 1998.

[10] Y. Hao, Q. Ye, Z. Pan, H. Cai, and R. Qu, "Analysis of spontaneous Brillouin scattering spectrum for different modulated pulse shape," Optik, vol. 124, no. 16, pp. 2417-2420, 2013.

[11] Y. Hao, Q. Ye, Z. Pan, H. Cai, and R. Qu, "Improvement of pulse shape on Brillouin optical time domain reflectometry," in OFS2012 22nd International Conference on Optical Fiber Sensors, vol. 8421 of Proceedings of SPIE, Beijing, China, October 2012. 
[12] H. Naruse and M. Tateda, "Launched pulse-shape dependence of the power spectrum of the spontaneous Brillouin backscattered light in an optical fiber," Applied Optics, vol. 39, no. 34, pp. 6376-6384, 2000.

[13] H. Naruse and M. Tateda, "Trade-off between the spatial and the frequency resolutions in measuring the power spectrum of the Brillouin backscattered light in an optical fiber," Applied Optics, vol. 38, no. 31, pp. 6516-6521, 1999.

[14] Y. Hao, Q. Ye, Z. Pan, H. Cai, R. Qu, and Z. Yang, "Effects of modulated pulse format on spontaneous Brillouin scattering spectrum and BOTDR sensing system," Optics \& Laser Technology, vol. 46, no. 1, pp. 37-41, 2013.

[15] Y. Yu, L. Luo, B. Li, L. Guo, J. Yan, and K. Soga, "Double peak-induced distance error in short-time-Fourier-transformBrillouin optical time domain reflectometers event detection and the recovery method," Applied Optics, vol. 54, no. 28, p. E196, 2015.

[16] I. Daubechies, "The wavelet transform, time-frequency localization and signal analysis," IEEE Transactions on Information Theory, vol. 36, no. 5, pp. 961-1005, 1990.

[17] E. Cordero and K. Grochenig, "Time-frequency analysis of localization operators," Journal of Functional Analysis, vol. 205, no. 1, pp. 107-131, 2003.

[18] J. B. Anderson, F. Rusek, and V. Owall, "Faster-than-Nyquist signaling," Proceedings of the IEEE, vol. 101, no. 8, pp. 1817-1830, 2013.

[19] Y. Li and L. J. Cimini Jr., "Bounds on the interchannel interference of OFDM in time-varying impairments," IEEE Transactions on Communications, vol. 49, no. 3, pp. 401-404, 2001.

[20] H. Weng and K. Lau, "Wavelets, period doubling, and timefrequency localization with application to organization of convection over the tropical western pacific," Journal of the Atmospheric Sciences, vol. 51, no. 17, pp. 2523-2541, 1994.

[21] L. Debnath, Wavelets and Signal Processing, Springer, Berlin, Germany, 2012.

[22] S. Chand, "Efficient time-frequency localization of a signal," International Journal of Digital Multimedia Broadcasting, vol. 2014, Article ID 529852, 10 pages, 2014.

[23] M. Niklès, L. Thévenaz, and P. A. Robert, "Brillouin gain spectrum characterization in single-mode optical fibers," Journal of Lightwave Technology, vol. 15, no. 10, pp. 1842-1851, 1997.

[24] R. W. Boyd, Nonlinear Optics, Academic Press, Amsterdam, The Netherlands, 3rd edition, 2008.

[25] G. Agrawal, Nonlinear Fibre Optics, Academic Press, 2nd edition, 1995.

[26] T. Horiguchi, K. Shimizu, T. Kurashima, M. Tateda, and Y. Koyamada, "Development of a distributed sensing technique using Brillouin scattering," Journal of Lightwave Technology, vol. 13, no. 7, pp. 1296-1302, 1995.

[27] S. Xie, M. Pang, X. Bao, and L. Chen, "Polarization dependence of Brillouin linewidth and peak frequency due to fiber inhomogeneity in single mode fiber and its impact on distributed fiber Brillouin sensing," Optics Express, vol. 20, no. 6, pp. 6385-6399, 2012.

[28] A. Minardo, R. Bernini, and L. Zeni, "Stimulated Brillouin scattering modeling for high-resolution, time-domain distributed sensing," Optics Express, vol. 15, no. 16, pp. 10397-10407, 2007.

[29] K. Nishiguchi, C.-H. Li, A. Guzik, and K. Kishida, "Synthetic spectrum approach for Brillouin optical time-domain reflectometry," Sensors, vol. 14, no. 3, pp. 4731-4754, 2014.
[30] D. Alasia, Advanced Trends in Nonlinear Opitcs Applied to Distributed Optical-Fibre Sensors, EPFL, Lausanne, Switzerland, 2006.

[31] T. Strohmer and S. Beaver, "Optimal OFDM design for timefrequency dispersive channels," IEEE Transactions on Communications, vol. 51, no. 7, pp. 1111-1122, 2003.

[32] R. Haas and J.-C. Belfiore, "A time-frequency well-localized pulse for multiple carrier transmission," Wireless Personal Communications, vol. 5, no. 1, pp. 1-18, 1997.

[33] F. Wang, W. Zhan, X. Zhang, and Y. Lu, "Improvement of spatial resolution for BOTDR by iterative subdivision method," Journal of Lightwave Technology, vol. 31, no. 23, pp. 3663-3667, 2013.

[34] H. Murayama, K. Kageyama, A. Shimada, and A. Nishiyama, "Improvement of spatial resolution for strain measurements by analyzing Brillouin gain spectrum," in 17th International Conference on Optical Fibre Sensors, vol. 5855 of Proceedings of SPIE, Bruges, Belgium, August 2005. 


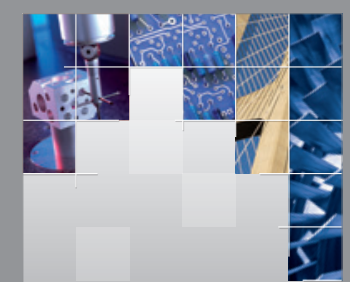

\section{Enfincering}
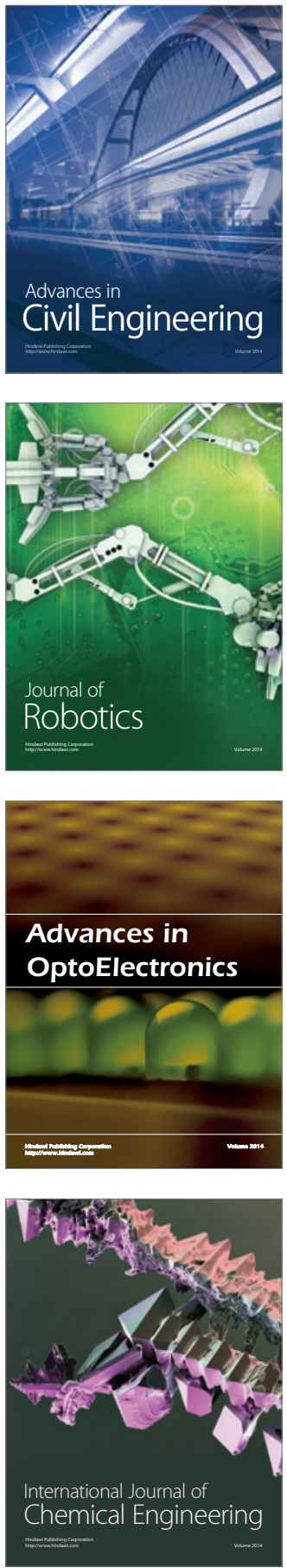

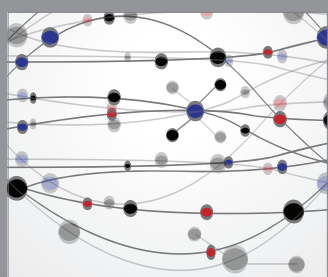

The Scientific World Journal

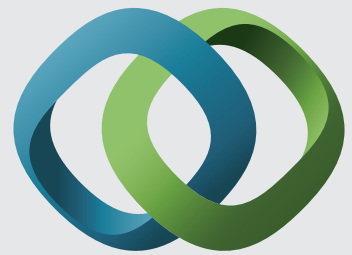

\section{Hindawi}

Submit your manuscripts at

http://www.hindawi.com
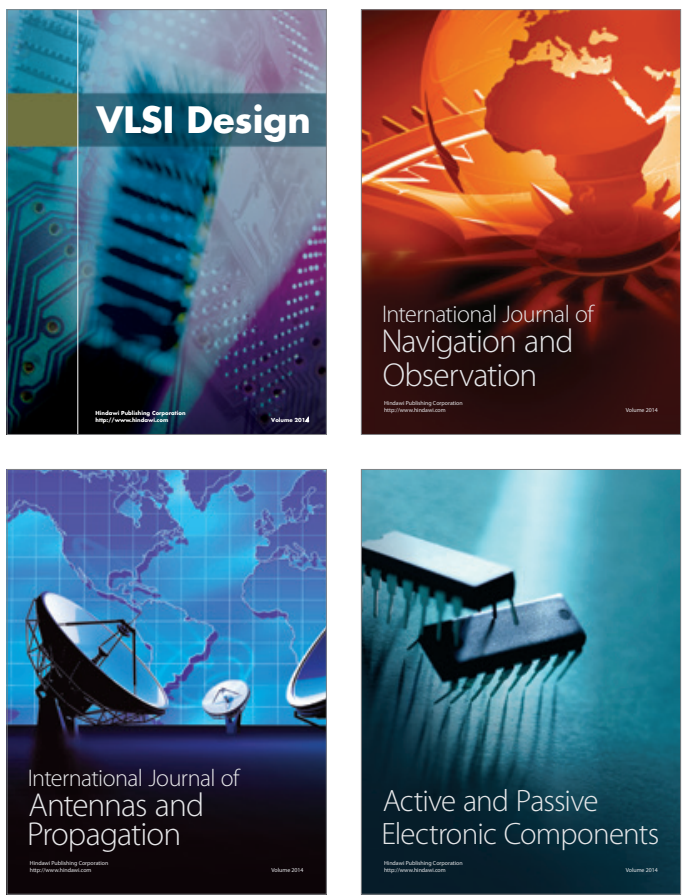
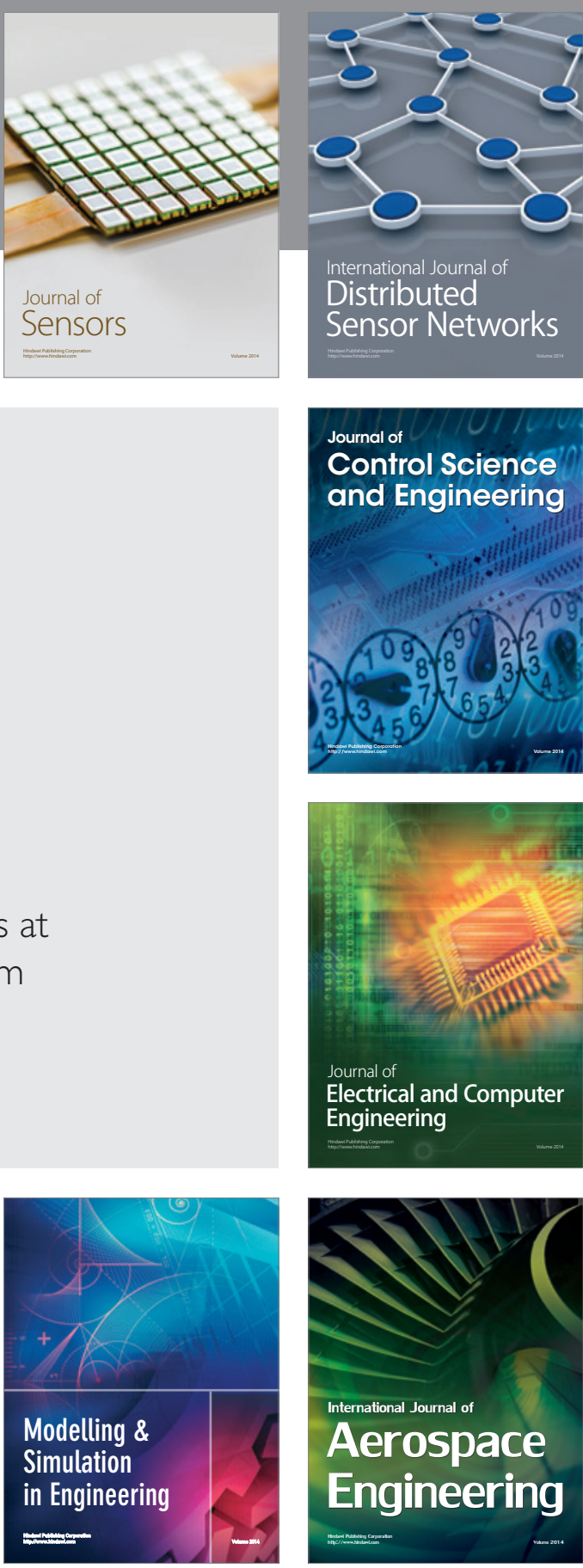

International Journal of

Distributed

Sensor Networks

Journal of

Control Science

and Engineering
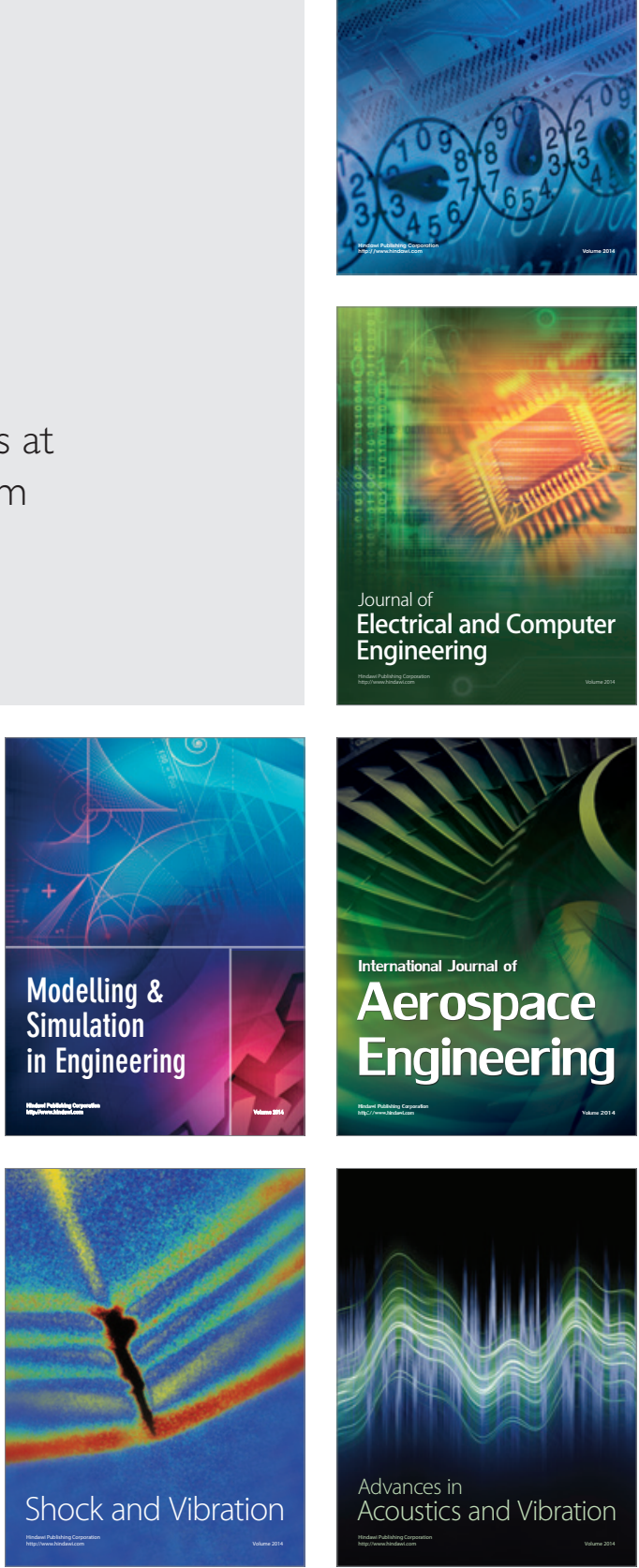